\title{
Aprendizaje cooperativo como aprendizaje efectivo
}

\author{
Ana González Sánchez y Eva Aguaded Ramírez. Universidad de Granada
}

Recepción: 29 de julio de 2015 | Revisión: 29 de julio de 2015 | Aceptación/Publicación: 30 de julio de 2015

Correspondencia: eaguaded@ugr.es

Citar: Gonzalez-Sanchez, A., \& Aguaded-Ramirez, E. (2015). Aprendizaje cooperativo como aprendizaje efectivo. ReiDoCrea, 4, 206212. http://hdl.handle.net/10481/37168

Resumen: En este trabajo se analiza la posible eficacia de una intervención educativa, dirigida a la mejora de las capacidades sociales y académicas del alumnado de Educación Primaria. Método: La muestra se compone de 14 alumnos y alumnas, de un aula de primer curso de Educación Primaria, pertenecientes a un centro de Granada. La investigación realizada se dividió en diferentes momentos; en primer lugar, se recogieron datos, previo a una posterior intervención educativa con carácter cooperativo, en la que se realizaron diversas acciones educativas. Posterior a dicha intervención, se recogió, nuevamente, información para ser contrastada con la obtenida anteriormente. Resultados: Los resultados obtenidos arrojaron ciertas mejoras en la participación, colaboración y rendimiento académico. Conclusiones: Necesidad de utilizar una metodología cooperativa para el alcance de un aprendizaje efectivo, cuando se trabaja con aprendizajes cooperativos.

Palabras clave: Aprendizaje Cooperativo | Comunidades de Aprendizaje

\section{COOPERATIVE LEARNING AS EFFECTIVE LEARNING}

\begin{abstract}
In this project we analyze the possible effectiveness of an educational intervention, directed to the improvement of social and academic skills for pupils in Primary Education. Method: The simple is composed of 14 pupils, male and female, in a 1st grade of a Primary Education classroom. The school is located in Granada. The research has been divided into different stages first; we collected all the information before an educational intervention in a cooperative way, in which we performed several educational actions. After this intervention, we collected the information again to be contrasted with the previously obtained. Result: The results show certain improvements in participation, teamwork and Educational achievement. Conclusions: Need for a cooperative approach for achieving effective learning, when working with cooperative learning.
\end{abstract}

Keywords: Cooperative Learning | Learning Communities

\section{Introducción}

Este trabajo se ha desarrollado, fundamentalmente, por la poca participación que tiene el alumnado, en su propio proceso de enseñanza-aprendizaje. A lo largo de los años, se ha puesto el énfasis al aprendizaje individual, competitivo e individualizado, en el que solo se han dado conceptos y no habilidades y destrezas, y mucho menos habilidades sociales, valores y actitudes (Ferreiro y Calderón, 2006).

Teniendo en cuenta la situación actual, en donde la educación tradicional no responde a las necesidades de la sociedad actual, es necesario, pues, un nuevo tipo de metodología, en el que esta, se adapte a la realidad del estudiante (Pérez y López, 2014). El ser humano vive en sociedad y es, por medio de la relación con sus iguales, por lo que crece y se desarrolla. Por lo cual, el uso de un método cooperativo en el que el alumnado se desarrolle y aprenda junto con sus iguales, es el factor clave para un aprendizaje significativo.

En base a diversas investigaciones, como las que aporta Vigotsky (2009), en donde se refleja que la sociedad es una pieza clave para el desarrollo del conocimiento, se establece como núcleo, en gran medida, la importancia de la relación del desarrollo cognitivo con el entorno sociocultural del alumnado, ya que, el aprendizaje viene dado por la interacción con el resto de personas, siendo, por tanto, el origen y el motor para el desarrollo intelectual, puesto que el alumnado tiene que interiorizar las experiencias. 
En las recientes aportaciones como las de Flecha (2002) se puede observar, cómo este asienta las bases del aprendizaje dialógico, de las Comunidades de Aprendizaje, en una metodología cooperativa, en la que los valores tanto de la cooperación, como los de la solidaridad, son fomentados en todo momento, puesto que, estas facilitan que todas las personas opten, en todo lo posible, por conseguir un aprendizaje necesario en la sociedad actual.

Es en este tipo de organización, como pueden ser las Comunidades de Aprendizaje, en las que se ve de primera mano, cómo, por medio de la cooperación, se va construyendo el conocimiento, a la vez que se adquieren otro tipo de habilidades y destrezas sociales. Esto, se debe, en gran parte, a la relación que existe entre el Aprendizaje cooperativo y el Aprendizaje dialógico, ya que este es un elemento de dicho Aprendizaje dialógico (Valls, 1999).

Por lo que el objetivo principal, es mostrar a los docentes, cómo, por medio de una metodología cooperativa, se puede conseguir un aprendizaje de calidad, aplicando dicha metodología no solo a las sesiones de Comunidades de Aprendizaje, sino al propio aula ordinaria. Ya que "Los poderosos efectos que tiene la cooperación sobre tantos aspectos distintos y relevantes determinan que el aprendizaje cooperativo se distinga de otros métodos de enseñanza y contribuya una de las herramientas más importantes para garantizar el buen rendimiento de los alumnos", (Johnson, Johnson y Holubec, 1999).

Fundamentando esto, además, en el Capítulo II, Artículo 8 del Decreto 97 del 13 de marzo del 2015 del Boletín Oficial de la Junta de Andalucía (BOJA), en la que se dice que la metodología debe partir de los intereses del alumno y además favorecer tanto al trabajo individual como cooperativo.

Además, dicha metodología ayudará a alcanzar las competencias clave, puesto que, como dice la Orden ECD/65/2015, de 21 de enero, por la que se describen las relaciones entre las competencias, los contenidos y los criterios de evaluación de la educación primaria, la educación secundaria obligatoria y el bachillerato, "Para un proceso de enseñanza-aprendizaje competencial las estrategias interactivas son las más adecuadas, al permitir compartir y construir el conocimiento y dinamizar la sesión de clase mediante el intercambio verbal y colectivo de ideas" (Pág. 7003). Además, de dicha Orden, se observa como esto es mencionado, a nivel autonómico, en Andalucía, tanto en el Decreto 97/2015, de 3 de marzo, por el que se establece la ordenación del currículo de la Educación Primaria en la Comunidad Autonómica de Andalucía, en su Artículo 6, donde se establecen dichas competencias claves, y, en la Orden de 17 marzo de 2015, por la que se desarrolla el currículo correspondiente a la Educación Primaria en Andalucía, en la que se determina qué competencias claves se deben alcanzan en cada una de las materias instrumentales.

En el centro, en el que se ha llevado a cabo el desarrollo del trabajo, ha sido aceptado el proyecto de Comunidades de Aprendizaje, para el curso 2015/2016. Este proyecto, ha tomado forma en este curso, siendo implantando a través del proyecto piloto desde enero de 2015. Esto supone, un cambio un tanto radical en la organización y metodología dentro del aula, pues la enseñanza tradicional queda obsoleta frente a este tipo de proyectos.

Por lo que, la necesidad principal, es demostrar cómo una metodología cooperativa, es la base principal para poder llegar a tener un proceso enseñanza-aprendizaje óptimo, cuando la finalidad, que se pretende es el desarrollo de aprendizajes activos y cooperativos, de forma cooperativa (De Miguel, 2006), como es el caso. 


\section{Método de investigación}

\section{Objetivos}

1. Analizar el comportamiento del alumnado a través de la recogida de información en las sesiones de Comunidades de Aprendizaje.

2. Modificar el comportamiento del alumnado a través de la intervención, por medio de acciones educativas, de carácter cooperativo.

3. Analizar el comportamiento del alumnado, después de la intervención por medio de acciones educativas de carácter cooperativo, a través de la recogida de información en las sesiones de Comunidades de Aprendizaje.

4. Analizar los cambios producidos, gracias a la intervención realizada.

\section{Población y muestra}

El centro, en el que se realiza la investigación, se encuentra situado en el Barrio de La Chana, en la periferia de la ciudad de Granada. La población sería todo el alumnado que compone el centro docente. Nosotros, nos vamos a basar en un muestreo accidental, por el que la muestra de nuestro trabajo, viene concebida por el criterio del alumnado del que tenemos acceso, por medio de las prácticas realizadas en el centro y con el grupo en cuestión.

La muestra de la investigación es, por tanto, un grupo de Educación Primaria, de primer curso. Esta aula cuenta con 14 alumnos, en los que 8 son niños y 6 son niñas.

\section{Procedimiento}

\section{Fases de la investigación}

1. Observación del comportamiento y rendimiento del alumnado y recogida de información.

2. Intervención por medio de acciones educativas de carácter cooperativo, en las materias instrumentales (matemáticas y lengua castellana).

3. Observación del comportamiento y rendimiento del alumnado y recogida de información, posterior a la intervención.

4. Análisis y contraste de los datos recogidos en las fases anteriores.

\section{Temporalización, espacio y organización}

El tiempo empleado ha sido aproximadamente de un mes. No ha sido preciso el uso de un tiempo prolongado, puesto que, no se ha pretendido generalizar, por tanto, no se ha considerado necesario realizar un análisis exhaustivo de la información. Teniendo por tanto un carácter más cualitativo que cuantitativo. Para el espacio, no ha sido preciso otro lugar, más que la propia aula, ya que, es en esta, donde se desarrollan tanto las clases, como las diversas sesiones de Comunidades de Aprendizaje. Para la organización del aula, el alumnado se ha repartido de manera grupal o en parejas. En esta organización de grupos y parejas, se ha intentado, en la mayor medida, que estos fueran lo más heterogéneos posibles.

\section{Diseño de la intervención}

En cuanto a los contenidos y objetivos curriculares, se han obtenido de la programación de aula. Pero, para poder llevar a cabo la intervención de una manera 
eficaz, se toma como base la infusión curricular. Dicha infusión curricular, tal y como dice Aguaded (2014):

"Es una modalidad de trabajo que nos permite el establecimiento de los contenidos referidos a la Acción Tutorial desde una perspectiva transversal en todo el currículo educativo, consiguiendo, si se hace de la forma adecuada, la integración de los aspectos tutoriales en una posición elevada dentro de las finalidades educativas explícitas en los planes formativos de los centros que así lo consideran" (Pág., 103)

Por lo que se han infusionado los objetivos, para conseguir una metodología cooperativa dentro del aula, junto con los objetivos que establece la programación de aula.

\section{Desarrollo de la intervención}

En un primer momento, desde la primera semana, se recogió información del alumnado a través de la observación, realizando esta recogida de datos por medio de un registro de observación. Esta recogida de datos, se ha efectuado en las sesiones de Comunidades de Aprendizaje. También, se han tenido en cuenta, la metodología y organización del profesorado del aula. Esta, era de carácter tradicional, en la que, aunque el alumnado, se organizara en parejas, trabajaba de forma individual.

En la segunda semana, se han realizado las diferentes acciones educativas con carácter cooperativo. Para ello, la intervención se vale de dos sesiones de materias instrumentales (matemáticas y lengua castellana). Dichas sesiones han estado repartidas a lo largo de la semana (lunes y miércoles), teniendo una duración de, aproximadamente, 55 minutos.

Llegando ya a la última fase de la intervención. En esta, se han tomado los mismos instrumentos de recogida que se utilizaron en la primera fase, para poder comprobar, junto con los datos que se han obtenido en la primera fase, si estos han llegado a alcanzar, una cierta mejoría con respecto a los que se obtuvieron en primer lugar.

Para terminar, se han contrastado y comparado los objetivos, y se han visto los resultados obtenidos.

\section{Instrumentos}

Para la recogida de datos en la intervención, se ha utilizado fundamentalmente la observación. Como instrumento, se han usado unos ítems, que han constituido un registro de observación, en los que se han señalado las diferentes intervenciones, que puede efectuar el alumnado, cuando trabaja en equipo, en las actividades presentadas en las sesiones de Comunidades de Aprendizaje.

\begin{tabular}{|ll|}
\hline \multicolumn{2}{|c|}{ Tabla 2. Registro de Observación } \\
\hline & Ítems \\
\hline 1 & Intervención individual, sin tener en cuenta las aportaciones de los demás. \\
2 & Interrupción o comentario fuera de la dinámica \\
3 & Explicación por parte del alumnado aventajado al menos aventajado. \\
4 & Competitividad por dar la respuesta antes que los compañeros \\
5 & Iniciativa para empezar a hacer las tareas \\
6 & Interés por acabar la tarea de manera grupal \\
7 & Interés por acabar la tarea de manera individual \\
8 & Negación a la hora de ayudar a los compañeros menos aventajados \\
9 & Búsqueda de aprobación o explicación por parte del profesorado o voluntario/a \\
10 & Dar simplemente la respuesta de la tarea o actividad, sin ningún tipo de explicación del contenido. \\
\hline
\end{tabular}




\section{Resultados}

Se puede observar cómo el alumnado, ha trabajado de manera más efectiva a la hora de realizar las actividades que se han programado en las sesiones de aprendizaje, terminando, en su mayoría las actividades que se habían planteado, en grupo. Han realizado más intervenciones significativas y se han ayudado los unos a los otros cuando se han presentado dudas o dificultades, sin necesidad de que, o el voluntariado o el profesorado, le incentiven a hacerlo, aunque sigue habiendo diversas intervenciones fuera de este tipo de dinámica, tales como interrupciones o comentarios que no tienen nada que ver con la actividad. Por otro lado, han trabajado de manera más autónoma y han precisado menos de la aprobación o explicación, por parte del profesorado o del voluntariado, aunque, siguen necesitando bastante ayuda por parte de ellos, puesto que no se han acostumbrado aún a trabajar con este tipo de organización y metodología.

La iniciativa por empezar a trabajar en las actividades, ha sufrido un gran aumento, puesto que se han visto más colaborativos, pero siguen, en cierta medida, queriendo acabar en primer lugar, por lo que la competitividad y el trabajo individual aún siguen persistiendo.

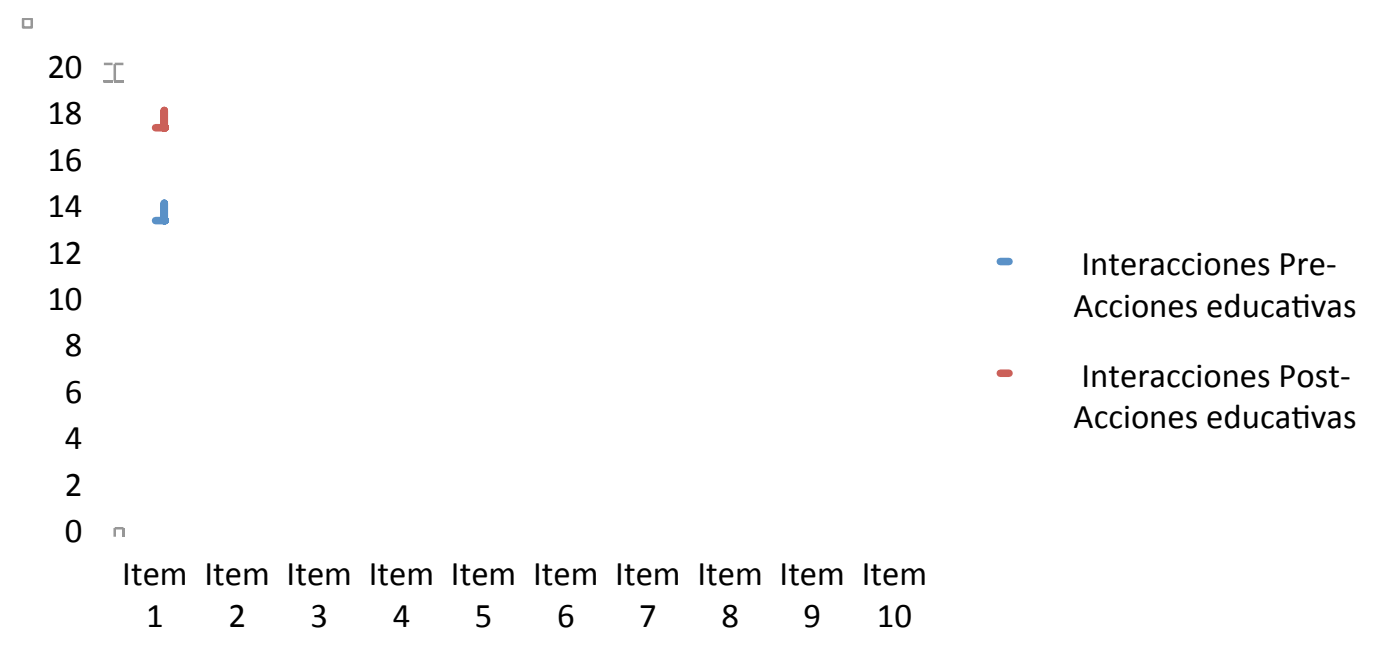

Figura 1. En esta figura se puede observar en el eje de coordenadas $Y$ el número de interacciones acometidas durante las sesiones de Comunidades de Aprendizaje. El eje de coordenadas $X$ expresa los ítems en los que se producen dichas interacciones.

\section{Discusión}

Como en todo tipo de investigaciones, se pueden encontrar diversos autores, que refuerzan, en cierta medida, esta investigación. En este caso, existen autores como Obeso, en Rodríguez, (2014), el cual, hace referencia a la metodología cooperativa, como una propuesta innovadora y destacable, para poder afrontar con éxito la llegada a España del Espacio Europeo de Educación Superior, que hace que se tengan que modificar las metodologías que se basan en clases magistrales, y tengan que adaptarse a clases más dinámicas en las que sea el propio alumnado, el participante de su propio aprendizaje. Pudiendo llevar esto a cabo, gracias a la metodología cooperativa por medio de actividades basadas en sucesos realistas, que hacen que la realidad se acerque al alumnado.

Por otro lado, se encuentran autores como Durán (2004), que corrobora que por medio de una modalidad de aprendizaje cooperativo, o una de sus vertientes, como 
puede ser la tutoría entre iguales, aun siendo esta poco explotada en la metodología docente, aporta al alumnado, no sólo beneficios a nivel académico, sino que, la interacción entre el propio grupo de iguales, teniendo este características distintas, se convierte en un elemento positivo, para poder alcanzar un aprendizaje significativo, eficaz y completo.

\section{Conclusiones}

Teniendo presentes los objetivos de la investigación, se puede ver cómo estos han sido alcanzados sin ningún problema, puesto que, por medio de las fases de la investigación, se han ido obteniendo, según iba desarrollándose dicha investigación e intervención. Tales objetivos han mostrado cómo, por medio de este tipo de metodologías, el alumnado tiende a intervenir más en las actividades que se desarrollan en el aula, al igual que dichas intervenciones son, cada vez, más significativas. Por lo que se puede concluir, que, por medio del alcance de los objetivos, obtienen unos resultados favorables.

Se ha de decir que los resultados obtenidos han sido en su mayor parte favorables para el alumnado. Por medio de este tipo de metodología, el alumnado trabaja e interviene en el aula de una forma constante y eficaz. Gracias a las acciones educativas, dicho alumnado se ha abierto más a la participación y a la colaboración con sus iguales, haciendo que no solo mejore a nivel individual, sino que el propio grupo avanza de un modo más óptimo.

Gracias a que, no solo se ha trabajado de una manera cooperativa en las sesiones de Comunidades de Aprendizaje, sino que se ha llevado a cabo este tipo de aprendizaje cooperativo en la propia metodología del aula, las mismas sesiones de Comunidades de Aprendizaje han resultado más fluidas y beneficiosas para los grupos, puesto que han sabido trabajar de forma más autónoma y sin necesidad de la intervención del profesorado, o la motivación del voluntariado.

De manera general, se podría decir que, si este tipo de metodología se implantara en la propia aula, tanto el ambiente de aprendizaje, como el nivel académico se elevarían. Además no solo se fomentaría el proceso de enseñanza-aprendizaje, sino que, las habilidades sociales y el clima del aula, sería los más adecuados para conseguir, el fin que se quiere alcanzar, es decir, para conseguir un aprendizaje eficaz.

\section{Referencias}

Aguaded, E. (2014). Procesos de planificación y desarrollo de la Acción Tutorial. En La Acción Tutorial en la educación actual. Madrid. Síntesis

De Miguel, M. (2006). Modalidades de enseñanza centradas en el desarrollo de competencias. Orientaciones para promover el cambio metodológico en el espacio europeo de educación. Oviedo. Universidad de Oviedo.

Decreto 97/2015, de 3 de marzo. Boletín Oficial de la Junta de Andalucía, №50, 2015, 13 de marzo.

Ferreiro, R., Calderón, M. (2006). El ABC del aprendizaje cooperativo. Trabajo en equipo para enseñar y aprender. Sevilla. Trillas

Flecha, R., \& Puigvert, L. (2002). Las comunidades de aprendizaje. Una propuesta por la igualdad educativa. Rexe,1(1),11-20. http://dialnet.unirioja.es/servlet/articulo?codigo=208325

Johnson, D., Johnson, R., Holubec, E. (1999). El aprendizaje cooperativo en el aula. Argentina. Paidas Educador.

Obeso, M. (2014). El aprendizaje cooperativo como metodología docente: una propuesta en una asignatura de posgrado. Nuevas metodologías didácticas. Madrid. ACCI. 
Orden 17 de marzo. Boletín Oficial de la Junta de Andalucía, №60, 2015, 27 de marzo.

Orden ECD/65/2015 de 21 de enero. Boletín Oficial del Estado, N²5, 2015, 29 de enero.

Pérez, R., López, R. (2015). El aprendizaje cooperativo. Una propuesta docente hacia el aprendizaje significativo de los estudiantes de la universidad. FUNCAS, http://www.funcas.es/publicaciones/Sumario.aspx?IdRef=7-05763

Valls, M (1999). Comunidades de aprendizaje dialógico para la sociedad de la información. Universidad de Barcelona.

Vigostky, L.S. (2009). El desarrollo de los procesos psicológicos superiores: interacción entre enseñanza y desarrollo. En: Estrategias de aprendizaje en la nueva universidad cubana. Cuba. Editorial Universitaria. 